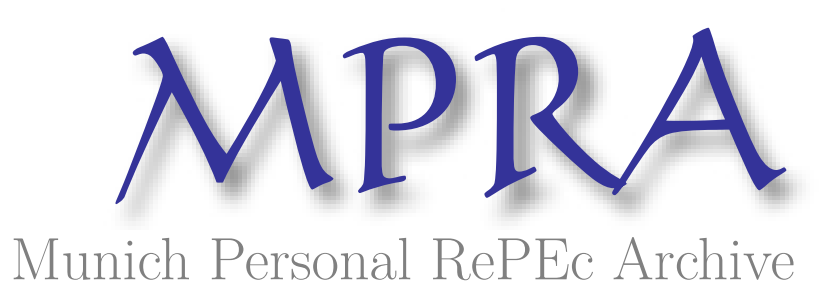

\title{
A note on positive semi-definiteness of some non-pearsonian correlation matrices
}

Mishra, SK

North-Eastern Hill University, Shillong (India)

14 June 2009

Online at https://mpra.ub.uni-muenchen.de/15725/

MPRA Paper No. 15725, posted 15 Jun 2009 05:51 UTC 


\section{A Note on Positive Semi-definiteness of Some Non-Pearsonian Correlation Matrices}

SK Mishra

Department of Economics North-Eastern Hill University Shillong, Meghalaya (India) mishrasknehu@yahoo.com

I. Introduction: A correlation matrix, $\mathfrak{R}$, is a real and symmetric $m \times m$ matrix such that $-1 \leq r_{i j} \in \mathfrak{R} \leq 1 ; i, j=1,2, \ldots, m$. Moreover, $r_{i i}=1$. The Pearsonian (or the product moment) correlation coefficient, e.g. $r_{12}$ (between two variates, say $x_{1}$ and $x_{2}$, each in $n$ observations), is given by the formula:

$$
r\left(x_{1}, x_{2}\right)=\operatorname{cov}\left(x_{1}, x_{2}\right) / \sqrt{\operatorname{var}\left(x_{1}\right) \cdot \operatorname{var}\left(x_{2}\right)}
$$

where, $\bar{x}_{a}=\frac{1}{n} \sum_{k=1}^{n} x_{k a} ; \operatorname{cov}\left(x_{1}, x_{2}\right)=\frac{1}{n} \sum_{k=1}^{n} x_{k 1} x_{k 2}-\bar{x}_{1}^{2} \bar{x}_{2}^{2}$ and $\operatorname{var}\left(x_{a}\right)=\operatorname{cov}\left(x_{a}, x_{a}\right) ; a=1,2$. A little of algebra also gives us the identity:

$$
r\left(x_{1}, x_{2}\right)=(1 / 4)\left[\operatorname{var}\left(x_{1}+x_{2}\right)-\operatorname{var}\left(x_{1}-x_{2}\right)\right] / \sqrt{\operatorname{var}\left(x_{1}\right) \cdot \operatorname{var}\left(x_{2}\right)}
$$

The Pearsonian correlation matrix is necessarily a positive semi-definite matrix (meaning that all its eigenvalues are non-negative) since it is the quadratic form of a real matrix, $X(n, m)$. It also implies that if $\mathfrak{R}$ is not a semi-positive matrix, then $X(n, m)$ is not a real matrix.

II. Robust Measures of Correlation: The Pearsonian coefficient of correlation as a measure of association between two variates is highly prone to the deleterious effects of outlier observations (data). Statisticians have proposed a number of formulas, other than the one that obtains Pearson's coefficient of correlation, that are considered to be less affected by errors of observation, perturbation or presence of outliers in the data. Some of them transform the variables, say $x_{1}$ and $x_{2}$, into $z_{1}=\phi_{1}\left(x_{1}\right)$ and $z_{2}=\phi_{2}\left(x_{2}\right)$, where $\phi_{a}\left(x_{a}\right)$ is a linear (or nonlinear) monotonic (order-preserving) rule of transformation or mapping of $x_{a}$ to $z_{a}$. Then, $r\left(z_{1}, z_{2}\right)$ is obtained by the appropriate formula and it is considered as a robust measure of $r\left(x_{1}, x_{2}\right)$. Some others use different measures of central tendency, dispersion and co-variation, such as median for mean, mean deviation for standard deviation and so on. In what follows, we present a few formulas of obtaining different types of correlation efficient.

II.1. Spearman's Rank Correlation Coefficient: If $x_{1}$ and $x_{2}$ are two variables, both in $n$ observations, and $z_{1}=\mathbb{R}\left(x_{1}\right)$ and $z_{2}=\mathbb{R}\left(x_{2}\right)$ are their rank numerals with $\mathbb{R}($.) as the rank-ordering rule, then the Pearson's formula applied on $\left(z_{1}, z_{2}\right)$ obtains the Spearman's correlation coefficient (Spearman, 1904). There is a simpler (but less general) formula that obtains rank correlation coefficient, given as:

$$
\rho\left(x_{1}, x_{2}\right)=r\left(z_{1}, z_{2}\right)=1-6 \sum_{k=1}^{n}\left(z_{k 1}-z_{k 2}\right)^{2} /\left[n\left(n^{2}-1\right)\right] \quad \ldots
$$


II.2. Signum Correlation Coefficient: Let $c_{1}$ and $c_{2}$ be the measures of central tendency or location (such as arithmetic mean or median) of $x_{1}$ and $x_{2}$ respectively. We transform them to $z_{k a}=\left(x_{k a}-c_{a}\right) /\left|x_{k a}-c_{a}\right|$ if $\left|x_{k a}-c_{a}\right|>0$, else $z_{k a}=1$. Then, $r\left(z_{1}, z_{2}\right)$ is the signum correlation coefficient (Blomqvist, 1950; Shevlyakov, 1997). Due to the special nature of transformation, we have

$$
r\left(z_{1}, z_{2}\right) \cong \operatorname{cov}\left(z_{1}, z_{2}\right)=(1 / n) \sum_{i=1}^{n} z_{i 1} z_{i 2}
$$

In this study we will use median as a measure of central tendency to obtain signum correlation coefficients.

II.3. Bradley's Absolute Correlation Coefficient: Bradley (1985) showed that if $\left(u_{k}, v_{k}\right) ; k=1, n$ are $n$ pairs of values such that the variables $u$ and $v$ have the same median $=0$ and the same mean deviation (from median) or $(1 / n) \sum_{k=1}^{n}\left|u_{k}\right|=(1 / n) \sum_{k=1}^{n}\left|v_{k}\right|=d \neq 0$, both of which conditions may be met by any pair of variables when suitably transformed, then the absolute correlation may be defined as

$$
\rho(u, v)=\sum_{k=1}^{n}\left(\left|u_{k}+v_{k}\right|-\left|u_{k}-v_{k}\right|\right) / \sum_{k=1}^{n}\left(\left|u_{k}\right|+\left|v_{k}\right|\right) .
$$

II.4. Shevlyakov Correlation Coefficient: Hampel et al. (1986) defined the median of absolute deviations (from median) as a measure of scale, $s_{H}\left(x_{a}\right)=\underset{k}{\operatorname{median}}\left|x_{k a}-\underset{k}{\operatorname{median}}\left(x_{k a}\right)\right| ; a=1,2$ which is a very robust measure of deviation, and using this measure, Shevlyakov (1997) defined median correlation,

$$
r_{\text {med }}=\left[\operatorname{med}^{2}|u|-\text { med }^{2}|v|\right] /\left[\text { med }^{2}|u|+\operatorname{med}^{2}|v|\right]
$$

where $u$ and $v$ are given as $u_{k}=\left(x_{k 1}-\operatorname{med}\left(x_{1}\right)\right) / s_{H}\left(x_{1}\right)+\left(x_{k 2}-\operatorname{med}\left(x_{2}\right)\right) / s_{H}\left(x_{2}\right)$ and $v_{k}=\left(x_{k 1}-\operatorname{med}\left(x_{1}\right)\right) / s_{H}\left(x_{1}\right)-\left(x_{k 2}-\operatorname{med}\left(x_{2}\right)\right) / s_{H}\left(x_{2}\right) ; k=1,2, \ldots, n$.

III. Are Robust Correlation Matrices Positive Semi-definite? In this study we investigate into the question whether the correlation matrix, $\mathfrak{R}$, whose any element $r_{i j}$ is a robust measure of correlation (obtained by the formulas such as Spearman's, Blomqvist's, Bradley's or Shevlyakov's), is positive semidefinite. We use the dataset given in Table-1 as the base data for our experiments.

We have carried out ten thousand experiments for each method (Spearman's $\rho$, Blomqvist's signum, Bradley's absolute $r$, and Shevlyakov's $r_{\text {med }}$ ) of computing robust correlation, $r_{i j} \in \mathfrak{R}$. In each experiment, the base data (Table-1) has been perturbed by a small (between -0.25 to 2.5) quantity generated randomly (and distributed uniformly) over $n$ observations on all the eight variables. In each experiment, the correlation matrix, $\mathfrak{R}$, has been computed and its eigenvalues are obtained. If any (at least one) eigenvalue of the correlation matrix has been found to be negative, the occurrence has been counted as a failure (of the matrix being positive semi-definite), else it is counted as a success. Such three sets of experiments have been carried out with three different seeds for generating random numbers (for perturbation).

IV. The results, Discussion and Conclusion: Our findings reveal that while Spearman's rho, Blomqvist's signum, and Bradley's absolute correlation formulas yield positive semi-definite correlation matrix (without any failure), the failure rate of Shevlyakov's formula is very high (about 81 percent: more exactly $81.47 \%, 80.94 \%$ and $81.41 \%$ for the three random number seeds: 13317,31921 and 17523 , 
respectively). Two sample correlation matrices (and their eigenvalues) are presented in Table-2.1 and Table-2.2. It is found that the smallest eigenvalue so often turns out to be negative.

The observed failure rate of Shevlyakov's correlation matrix raises a question whether it can be used directly for further analysis of correlation matrices - without being approximated by the nearest positive semi-definite matrix (Mishra, 2008). While the product moment correlation coefficient (of Karl Pearson) is so much sensitive to the outliers, the robust nature of Shevlyakov's correlation coefficient is attractive. But, unfortunately, its robustness goes along with its being extremely prone to non-positive semi-definiteness and unsuitability to multivariate analysis. In view of these findings, it is safe to use Spearman's $\rho$, Blomqvist's signum or Bradley's absolute $r$ rather than Shevlyakov's correlation coefficient for constructing correlation matrices for any further analysis.

\begin{tabular}{|r|r|r|r|r|r|r|r|r|r|r|r|r|r|r|r|r|r|}
\hline \multicolumn{10}{|c|}{ Table-1: Base Dataset for Computation of Robust Correlation Matrices by Different Methods } \\
\hline SL. No. & $x_{1}$ & $x_{2}$ & $x_{3}$ & $x_{4}$ & $x_{5}$ & $x_{6}$ & $x_{7}$ & $x_{8}$ & SL. No. & $x_{1}$ & $x_{2}$ & $x_{3}$ & $x_{4}$ & $x_{5}$ & $x_{6}$ & $x_{7}$ & $x_{8}$ \\
\hline 1 & 18 & 3 & 1 & 2 & 3 & 3 & 2 & 7 & 20 & 8 & 2 & 9 & 4 & 9 & 11 & 7 & 2 \\
\hline 2 & 17 & 17 & 6 & 6 & 13 & 10 & 16 & 16 & 21 & 13 & 3 & 9 & 1 & 7 & 5 & 3 & 9 \\
\hline 3 & 6 & 1 & 6 & 1 & 10 & 16 & 5 & 7 & 22 & 15 & 4 & 7 & 5 & 6 & 15 & 12 & 15 \\
\hline 4 & 5 & 4 & 2 & 1 & 3 & 2 & 6 & 1 & 23 & 17 & 16 & 11 & 5 & 9 & 10 & 10 & 12 \\
\hline 5 & 21 & 10 & 8 & 3 & 7 & 7 & 12 & 8 & 24 & 2 & 7 & 5 & 1 & 3 & 1 & 1 & 1 \\
\hline 6 & 14 & 20 & 7 & 3 & 14 & 16 & 19 & 14 & 25 & 3 & 7 & 3 & 1 & 2 & 12 & 4 & 8 \\
\hline 7 & 3 & 15 & 12 & 1 & 2 & 15 & 10 & 9 & 26 & 20 & 19 & 4 & 4 & 8 & 13 & 14 & 10 \\
\hline 8 & 4 & 13 & 1 & 1 & 1 & 6 & 8 & 6 & 27 & 19 & 18 & 6 & 2 & 16 & 14 & 19 & 12 \\
\hline 9 & 18 & 16 & 4 & 1 & 1 & 4 & 5 & 5 & 28 & 3 & 14 & 9 & 3 & 11 & 5 & 10 & 3 \\
\hline 10 & 4 & 14 & 8 & 4 & 3 & 16 & 12 & 14 & 29 & 8 & 2 & 7 & 1 & 10 & 4 & 2 & 1 \\
\hline 11 & 17 & 14 & 8 & 9 & 15 & 11 & 20 & 13 & 30 & 16 & 21 & 11 & 9 & 10 & 18 & 18 & 17 \\
\hline 12 & 3 & 9 & 4 & 6 & 4 & 4 & 4 & 6 & 31 & 5 & 10 & 4 & 3 & 12 & 2 & 11 & 6 \\
\hline 13 & 7 & 5 & 5 & 2 & 12 & 9 & 13 & 10 & 32 & 21 & 17 & 9 & 8 & 11 & 13 & 15 & 10 \\
\hline 14 & 12 & 6 & 6 & 3 & 2 & 8 & 9 & 8 & 33 & 14 & 8 & 4 & 3 & 5 & 6 & 10 & 13 \\
\hline 15 & 1 & 5 & 3 & 4 & 12 & 15 & 12 & 11 & 34 & 9 & 6 & 1 & 1 & 2 & 10 & 8 & 4 \\
\hline 16 & 11 & 1 & 7 & 1 & 3 & 2 & 3 & 1 & 35 & 19 & 16 & 7 & 2 & 1 & 6 & 7 & 9 \\
\hline 17 & 9 & 12 & 6 & 8 & 12 & 16 & 20 & 16 & 36 & 19 & 15 & 10 & 7 & 4 & 17 & 17 & 15 \\
\hline 18 & 16 & 5 & 3 & 1 & 6 & 3 & 3 & 7 & 37 & 22 & 5 & 7 & 1 & 6 & 3 & 4 & 6 \\
\hline 19 & 10 & 11 & 7 & 10 & 8 & 14 & 13 & 15 & Note: This dataset has been perturbed in our experiments \\
\hline
\end{tabular}

Table-2.1. Shevlyakov's Robust Correlation matrix and its Eigenvalues (Sample-1)

\begin{tabular}{|c|c|c|c|c|c|c|c|c|}
\hline Variable & $x_{1}$ & $x_{2}$ & $x_{3}$ & $x_{4}$ & $x_{5}$ & $x_{6}$ & $x_{7}$ & $x_{8}$ \\
\hline$x_{1}$ & 1.00000 & 0.59053 & 0.27080 & 0.13168 & 0.20532 & 0.10974 & 0.41999 & 0.40193 \\
\hline$x_{2}$ & 0.59053 & 1.00000 & 0.34953 & 0.65555 & 0.56049 & 0.58320 & 0.85656 & 0.66425 \\
\hline$x_{3}$ & 0.27080 & 0.34953 & 1.00000 & 0.19778 & 0.21678 & 0.22393 & 0.25129 & 0.34782 \\
\hline$x_{4}$ & 0.13168 & 0.65555 & 0.19778 & 1.00000 & 0.47509 & 0.66117 & 0.83071 & 0.60398 \\
\hline$x_{5}$ & 0.20532 & 0.56049 & 0.21678 & 0.47509 & 1.00000 & 0.67361 & 0.67501 & 0.39314 \\
\hline$x_{6}$ & 0.10974 & 0.5 & 0.2 & 0.6 & 61 & 000 & 0.79762 & 0.70722 \\
\hline$x_{7}$ & 0.41999 & 0.85656 & 0.25129 & 0.83071 & 0.67501 & 0.79762 & 1.00000 & 0.74020 \\
\hline$x_{8}$ & 0.40193 & 0.66425 & 0.34782 & 0.60398 & 0.39314 & 0.70722 & 0.74020 & 1.00000 \\
\hline \multicolumn{9}{|c|}{ Eigenvalues (in descending order) } \\
\hline & 4.62370 & 1.17033 & 0.79635 & 0.61840 & 0.42434 & 0.16789 & 0.14943 & 0.04956 \\
\hline
\end{tabular}




\begin{tabular}{|c|c|c|c|c|c|c|c|c|}
\hline Variable & $x_{1}$ & $x_{2}$ & $x_{3}$ & $x_{4}$ & $x_{5}$ & $x_{6}$ & $x_{7}$ & $x_{8}$ \\
\hline$x_{1}$ & 1.00000 & 0.58154 & 0.36318 & 0.28763 & 0.20059 & -0.01382 & 0.43710 & 0.45526 \\
\hline$x_{2}$ & 0.58154 & 1.00000 & 0.48691 & 0.65733 & 0.46806 & 0.50631 & 0.86147 & 0.63320 \\
\hline$x_{3}$ & 0.36318 & 0.48691 & 1.00000 & 0.32810 & 0.17847 & 0.44367 & 0.23434 & 0.43348 \\
\hline$x_{4}$ & 0.28763 & 0.65733 & 0.32810 & 1.00000 & 0.42870 & 0.60102 & 0.85698 & 0.62314 \\
\hline$x_{5}$ & 0.20059 & 0.46806 & 0.17847 & 0.42870 & 1.00000 & 0.61081 & 0.67040 & 0.41083 \\
\hline$x_{6}$ & -0.01382 & 0.50631 & 0.44367 & 0.60102 & 0.61081 & 1.00000 & 0.86103 & 0.73863 \\
\hline$x_{7}$ & 0.43710 & 0.86147 & 0.23434 & 0.85698 & 0.67040 & 0.86103 & 1.00000 & 0.69714 \\
\hline$x_{8}$ & 0.45526 & 0.63320 & 0.43348 & 0.62314 & 0.41083 & 0.73863 & 0.69714 & 1.00000 \\
\hline \multicolumn{9}{|c|}{ Eigenvalues (in descending order) } \\
\hline & 4.67015 & 1.20149 & 0.83475 & 0.58967 & 0.42884 & 0.25716 & 0.13572 & -0.11778 \\
\hline
\end{tabular}

\section{References}

- Blomqvist, N. (1950) "On a Measure of Dependence between Two Random Variables", Annals of Mathematical Statistics, 21(4): 593-600.

- Bradley, C. (1985) “The Absolute Correlation”, The Mathematical Gazette, 69(447): 12-17.

- Hampel, F. R., Ronchetti, E.M., Rousseeuw, P.J. and W. A. Stahel, W.A. (1986) Robust Statistics: The Approach Based on Influence Functions, Wiley, New York.

- Mishra, S.K. (2008) "The Nearest Correlation Matrix Problem: Solution by Differential Evolution Method of Global Optimization", Journal of Quantitative Economics, New Series, 6(1\&2): 240262.

- Shevlyakov, G.L. (1997) "On Robust Estimation of a Correlation Coefficient", Journal of Mathematical Sciences, 83(3): 434-438.

- Spearman, C. (1904) "The Proof and Measurement of Association between Two Things", American Journal of Psychology, 15: 88-93. 


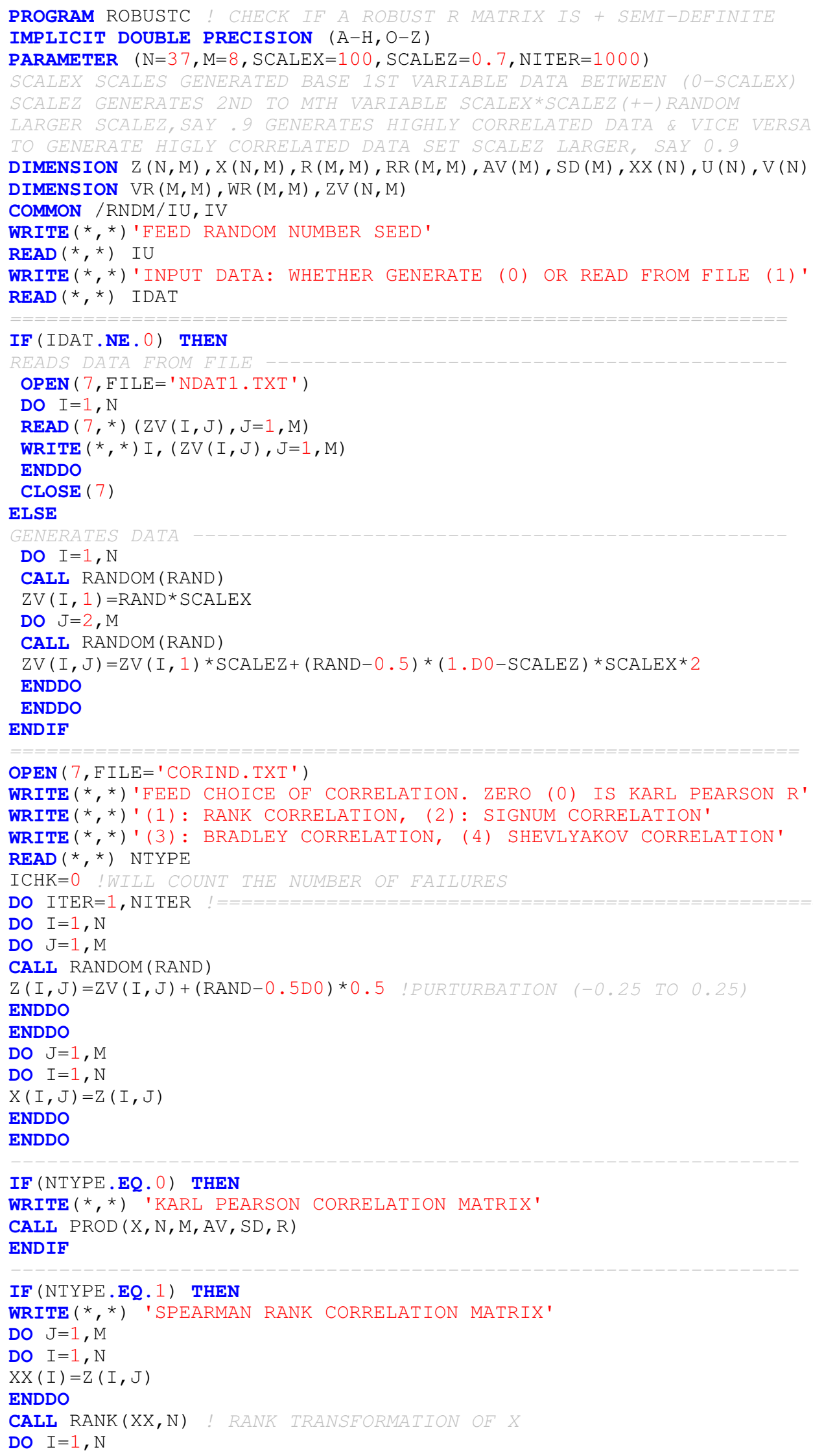




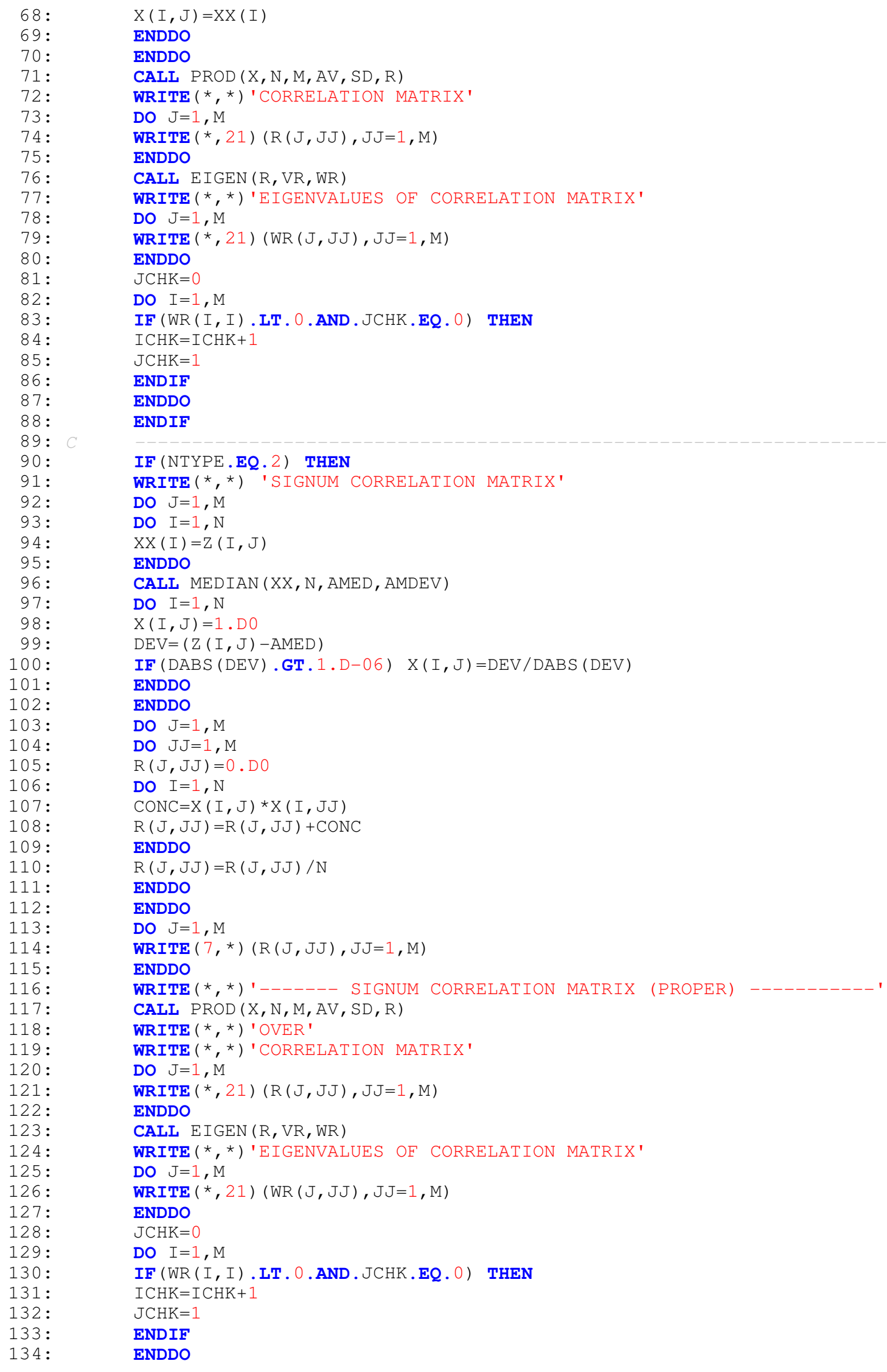




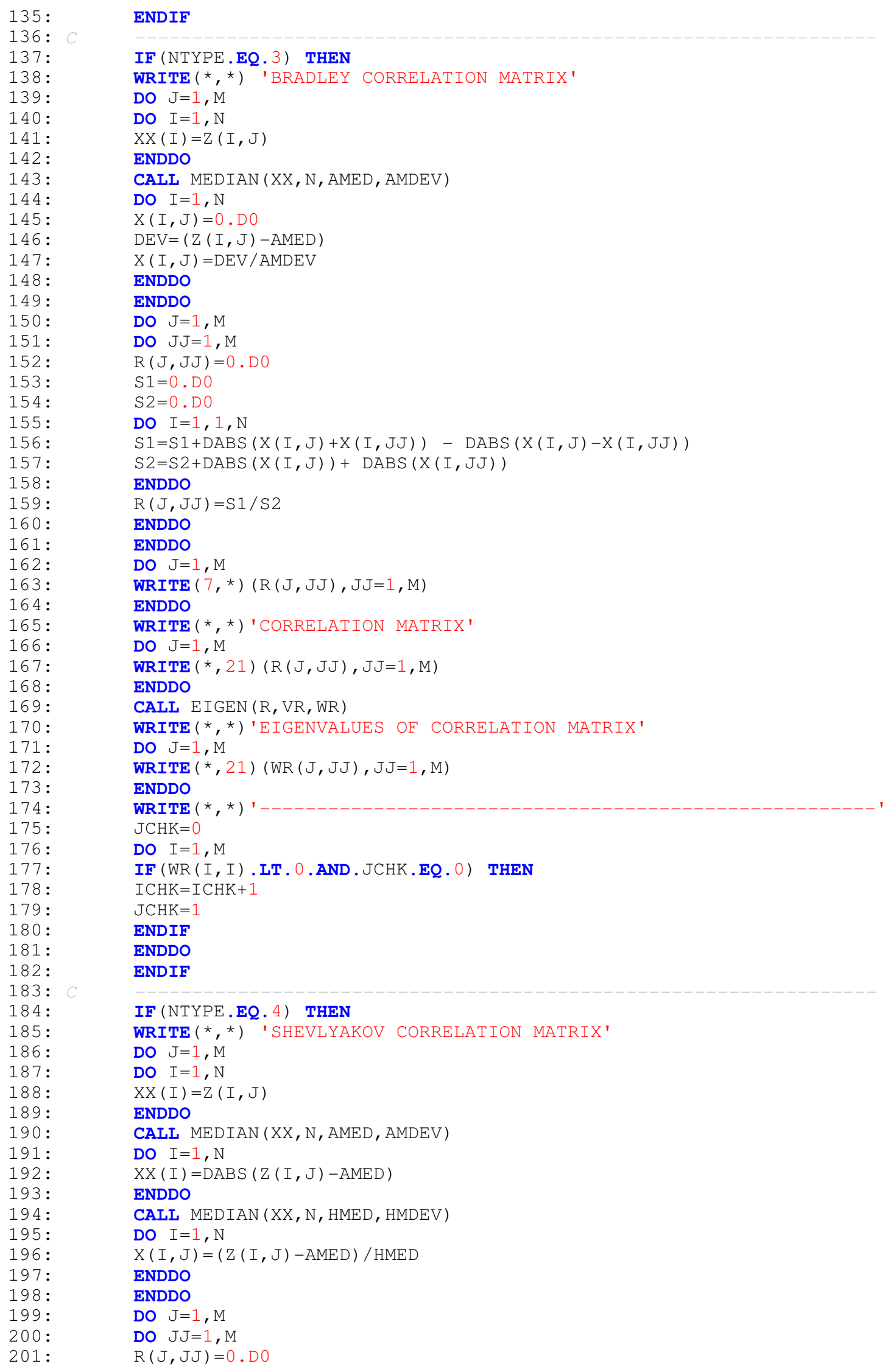


202:

203:

204 :

205:

206 :

207:

208:

209:

210:

211:

212:

213:

214 :

215:

216:

217:

218:

219:

220 :

221:

222:

223 :

224 :

$225:$

226:

227:

228:

229:

230 :

231:

232:

233:

234 :

235:

236:

237:

238:

239:

240 :

241:

242:

243:

244 :

$245:$

246 :

247 :

248:

249:

250 :

251:

252:

253:

254 :

255:

256:

257:

258:

259:

260:

261:

262:

$263:$

$264:$

265:

$266:$

267:

268:
DO $I=1, N$

$\mathrm{U}(\mathrm{I})=\operatorname{DABS}(X(I, J)+X(I, J J))$

$V(I)=\operatorname{DABS}(X(I, J)-X(I, J J))$

ENDDO

CALL MEDIAN (U, N, UMED, UMDEV)

CALL $\operatorname{MEDIAN}(V, N, \operatorname{VMED}, \operatorname{VMDEV})$

$\mathrm{R}(\mathrm{J}, \mathrm{J} J)=(\mathrm{UMED} * * 2-\operatorname{VMED} * * 2) /(\mathrm{UMED} * \star 2+\operatorname{VMED} * \star 2)$

ENDDO

ENDDO

WRITE (*,*) 'CORRELATION MATRIX'

DO $\mathrm{J}=1, \mathrm{M}$

WRITE $(*, 21)(R(J, J J), J J=1, M)$

ENDDO

CALL EIGEN (R, VR, WR)

WRITE (*,*) 'EIGENVALUES OF CORRELATION MATRIX'

DO $\mathrm{J}=1, \mathrm{M}$

WRITE (*,21) (WR ( J, JJ ), J J =1, M)

ENDDO

$\operatorname{WRITE}(*, *)$

$\mathrm{JCHK}=0$

DO $I=1, M$

IF (WR ( I, I ) .IT. O . AND.JCHK.EQ. O) THEN

$I C H K=I C H K+1$

$\mathrm{JCHK}=1$

ENDIF

ENDDO

ENDIF

FORMAT (10F 7.3)

21 FORMAT (10F 8.5)

ENDDO

WRITE (*,*) 'PERCENT OF (-) EIGENVALUE CASES=', ICHK/FLOAT (NITER) *100 CLOSE ( 7 )

END

SUBROUTINE PROD $(X, N, M, A V, S D, R)$

IMPLICIT DOUBLE PRECISION (A-H,O-Z)

DIMENSION $X(N, M), R(M, M), A V(M), S D(M)$

DO $J=1, M$

$\mathrm{AV}(\mathrm{J})=0 . \mathrm{D} 0$

$\mathrm{SD}(\mathrm{J})=0 \cdot \mathrm{D} 0$

DO $I=1, N$

$\mathrm{AV}(\mathrm{J})=\mathrm{AV}(\mathrm{J})+\mathrm{X}(\mathrm{I}, \mathrm{J})$

$\operatorname{SD}(J)=\operatorname{SD}(J)+X(I, J) * \star 2$

ENDDO

$\operatorname{SD}(J)=\operatorname{DSQRT}((N * \operatorname{SD}(J)-\operatorname{AV}(J) * A V(J)) / N * \star 2)$

$\mathrm{AV}(\mathrm{J})=\mathrm{AV}(\mathrm{J}) / \mathrm{N}$

ENDDO

DO $\mathrm{J}=1, \mathrm{M}$

DO $J J=1, M$

$R(J, J J)=0$. D 0

DO $I=1, N$

$R(J, J J)=R(J, J J)+X(I, J) * X(I, J J)$

ENDDO

$R(J, J J)=(R(J, J J) / N-A V(J) * A V(J J)) /(S D(J) * S D(J J))$

ENDDO

ENDDO

DO $J=1, M$

$\operatorname{WRITE}(7, *)(R(J, J J), J J=1, M)$

ENDDO

WRITE $(*, 1)(\mathrm{AV}(\mathrm{J}), \mathrm{J}=1, \mathrm{M})$

$\operatorname{WRITE}(*, 1)(\mathrm{SD}(\mathrm{J}), \mathrm{J}=1, \mathrm{M})$

1 FORMAT ( 8F 9.5$)$

$\operatorname{WRITE}(*, *)$ '

RETURN

END 


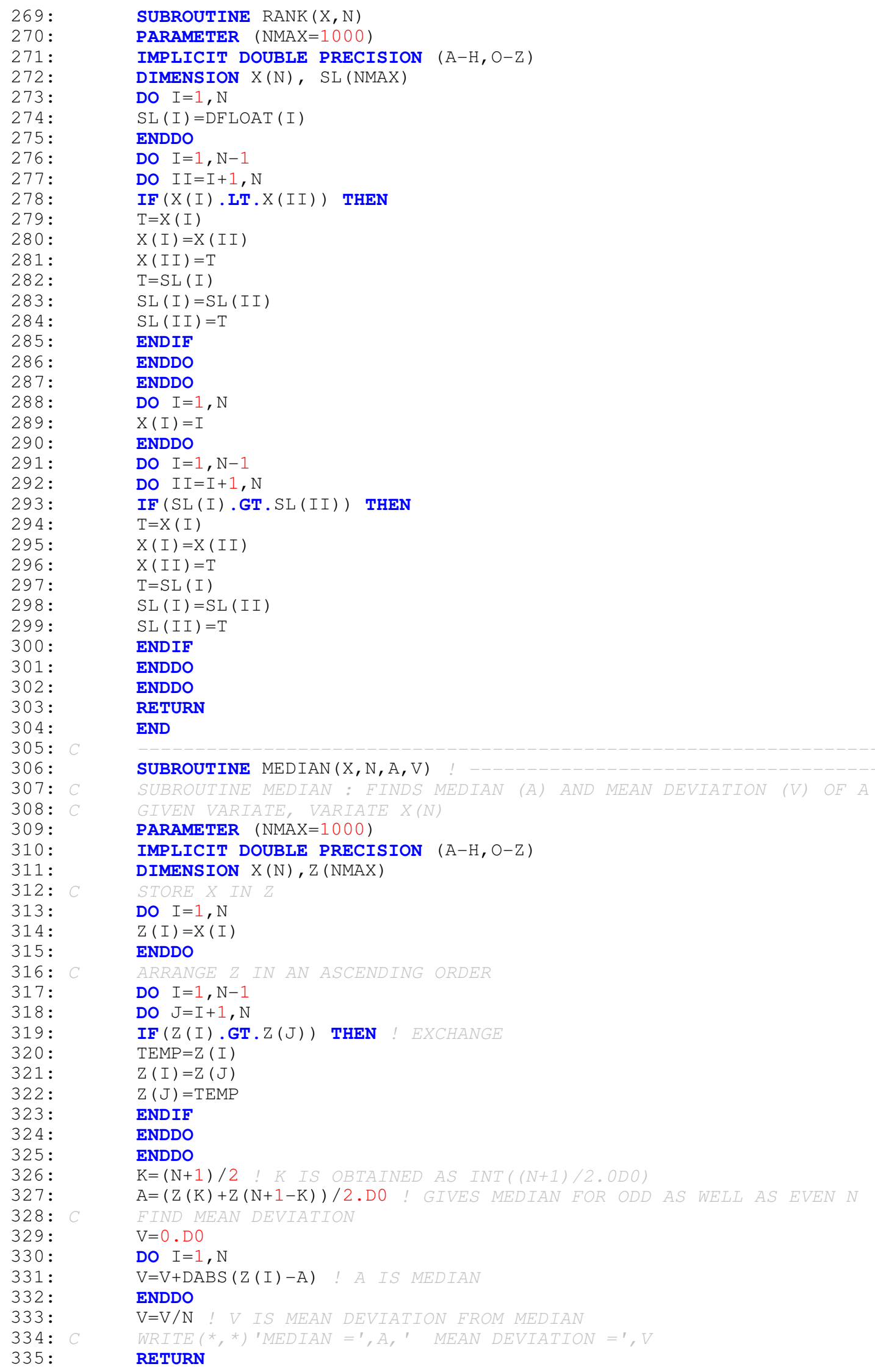




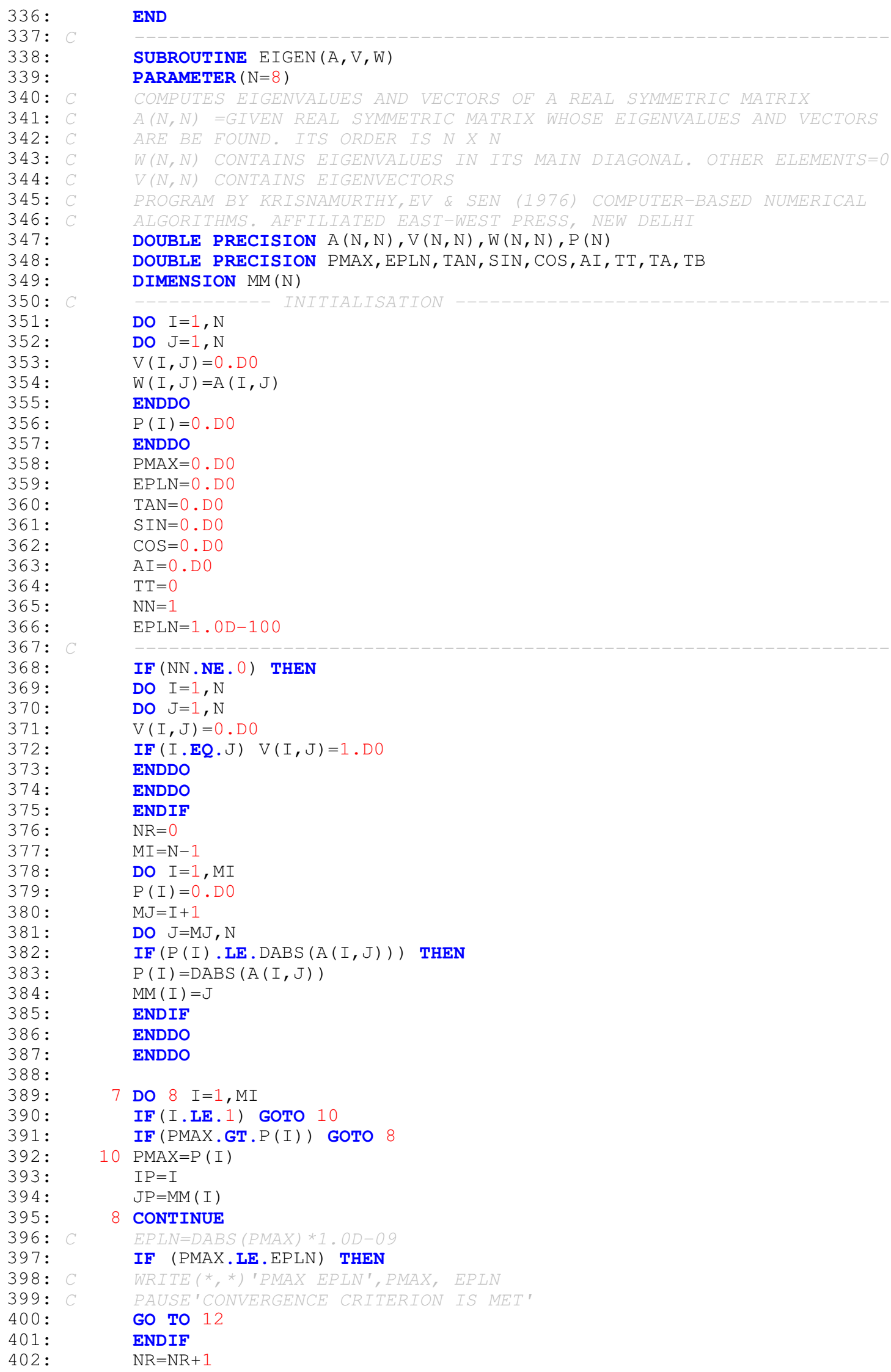


403:

404 :

405:

406:

407:

408:

409:

410 :

411:

412:

413:

414:

415:

416:

417:

418:

419:

420 :

421:

422:

423:

424 :

425:

426 :

427:

428:

429:

430 :

431 :

432:

433:

434 :

435:

436 :

437:

438:

439:

440 :

441 :

442:

443 :

444 :

445 :

446 :

447:

448:

449:

450 :

451:

452:

453:

454 :

455:

456 :

457:

458:

459:

460:

461:

462:

$463:$

$464:$

$465:$

466:

$467:$

468:

469:
$\mathrm{TA}=2 . \mathrm{D} 0 * \mathrm{~A}(\mathrm{IP}, \mathrm{JP})$

$\mathrm{TB}=(\mathrm{DABS}(\mathrm{A}(\mathrm{IP}, \mathrm{IP})-\mathrm{A}(\mathrm{JP}, \mathrm{JP}))+$

$\operatorname{IDSQRT}((\mathrm{A}(\mathrm{IP}, \mathrm{IP})-\mathrm{A}(\mathrm{JP}, \mathrm{JP})) \star \star 2+4 . \mathrm{D} 0 * \mathrm{~A}(\mathrm{IP}, \mathrm{JP}) \star \star 2))$

$\mathrm{TAN}=\mathrm{TA} / \mathrm{TB}$

IF (A (IP, IP ). LT.A (JP, JP) ) TAN=-TAN

$\mathrm{COS}=1 . \mathrm{D} 0 / \mathrm{DSQRT}(1 \cdot \mathrm{D} 0+\mathrm{TAN} * \star 2)$

$\mathrm{SIN}=\mathrm{TAN} * \mathrm{COS}$

$\mathrm{A} I=\mathrm{A}(\mathrm{IP}, \mathrm{IP})$

$A(I P, I P)=(C O S * \star 2) *(A I+T A N *(2 . D 0 * A(I P, J P)+T A N * A(J P, J P)))$

$A(J P, J P)=(C O S * \star 2) *(A(J P, J P)-T A N *(2 . D 0 * A(I P, J P)-T A N * A I))$

$A(I P, J P)=0 . D 0$

IF ( $A(I P, I P) . G E . A(J P, J P))$ GO TO 15

$\mathrm{TT}=\mathrm{A}(\mathrm{IP}, \mathrm{IP})$

$\mathrm{A}(\mathrm{IP}, \mathrm{IP})=\mathrm{A}(\mathrm{JP}, \mathrm{JP})$

$A(J P, J P)=T T$

IF (SIN.GE.O.DO) GO TO 16

$\mathrm{TT}=\mathrm{COS}$

GO TO 17

$16 \mathrm{TT}=-\mathrm{COS}$

$17 \operatorname{COS}=\operatorname{DABS}(\mathrm{SIN})$

$\mathrm{SIN}=\mathrm{TT}$

15 DO $18 \mathrm{I}=1, \mathrm{MI}$

IF ( I-IP) 19, 18, 20

20 IF ( I.EQ.JP) GO TO 18

19 IF (MM(I) .EQ. IP) GO TO 21

IF (MM (I) .NE.JP) GO TO 18

$21 \mathrm{~K}=\mathrm{MM}(\mathrm{I})$

$\mathrm{TT}=\mathrm{A}(\mathrm{I}, \mathrm{K})$

$A(I, K)=0 . D 0$

$\mathrm{MJ}=\mathrm{I}+1$

$P(I)=0 . D 0$

DO $22 \mathrm{~J}=\mathrm{MJ}, \mathrm{N}$

IF (P(I).GT.DABS (A(I, J ))) GO TO 22

$P(I)=\operatorname{DABS}(A(I, J))$

$\mathrm{MM}(\mathrm{I})=\mathrm{J}$

22 CONTINUE

$A(I, K)=T T$

18 CONTINUE

$P(I P)=0 . D 0$

$\mathrm{P}(\mathrm{JP})=0 . \mathrm{D} 0$

DO $23 \mathrm{I}=1, \mathrm{~N}$

IF (I-IP) 24, 23, 25

$24 \mathrm{TT}=\mathrm{A}(\mathrm{I}, \mathrm{IP})$

$A(I, I P)=C O S * T T+S I N * A(I, J P)$

IF (P(I).GE.DABS (A (I, IP ))) GO TO 26

$P(I)=\operatorname{DABS}(A(I, I P))$

$\mathrm{MM}(\mathrm{I})=\mathrm{IP}$

$26 A(I, J P)=-S I N * T T+C O S * A(I, J P)$

IF (P(I).GE.DABS (A (I, JP))) GO TO 23

$30 \mathrm{P}(\mathrm{I})=\mathrm{DABS}(\mathrm{A}(\mathrm{I}, \mathrm{JP}))$

$\mathrm{MM}(\mathrm{I})=\mathrm{JP}$

GO TO 23

25 IF(I.LT.JP) GO TO 27

IF (I.GT.JP) GO TO 28

IF (I.EQ.JP) GO TO 23

$27 \mathrm{TT}=\mathrm{A}(\mathrm{IP}, \mathrm{I})$

$A(I P, I)=C O S \star T T+S I N * A(I, J P)$

IF (P(IP). GE.DABS (A(IP, I )) ) GO TO 29

$P(I P)=\operatorname{DABS}(A(I P, I))$

$\mathrm{MM}(\mathrm{IP})=\mathrm{I}$

$29 A(I, J P)=-T T * S I N+C O S * A(I, J P)$

IF (P(I).GE.DABS (A (I, JP))) GO TO 23

GO TO 30

$28 \mathrm{TT}=\mathrm{A}(\mathrm{IP}, \mathrm{I})$

$\mathrm{A}(\mathrm{IP}, \mathrm{I})=\mathrm{TT} * \mathrm{COS}+\mathrm{SIN} * \mathrm{~A}(\mathrm{JP}, \mathrm{I})$

IF $(P(I P) . G E . D A B S(A(I P, I)))$ Go TO 31

$P(I P)=\operatorname{DABS}(A(I P, I))$ 
470 :

$471:$

$472:$

$473:$

$474:$

$475:$

476 :

$477:$

$478:$

479:

480 :

$481:$

482 :

$483:$

$484:$

$485:$

$486:$

$487:$

488:

489:

490:

491:

492:

493:

494:

$495:$

496:

$497:$

$498:$

499:

500 :

$501:$

$502:$

$503:$

$504:$

$505:$

$506:$

$507:$

508:

$\mathrm{MM}(\mathrm{IP})=\mathrm{I}$

$31 \mathrm{~A}(\mathrm{JP}, \mathrm{I})=-\mathrm{TT} * \mathrm{SIN}+\mathrm{COS} * \mathrm{~A}(\mathrm{JP}, \mathrm{I})$

IF (P (JP) . GE.DABS (A (JP, I )) ) GO TO 23

$P(J P)=D A B S(A(J P, I))$

$\mathrm{MM}(\mathrm{JP})=\mathrm{I}$

23 CONTINUE

IF (NN.EQ.0) GOTO 7

DO $32 I=1, N$

$\mathrm{TT}=\mathrm{V}(\mathrm{I}, \mathrm{IP})$

$\mathrm{V}(\mathrm{I}, \mathrm{IP})=\mathrm{TT}$ *COS $+\mathrm{SIN} * \mathrm{~V}(\mathrm{I}, \mathrm{JP})$

$V(I, J P)=-T T * S I N+C O S * V(I, J P)$

32 CONTINUE

GO TO 7

12 DO $\mathrm{I}=1, \mathrm{~N}$

$P(I)=A(I, I)$

ENDDO

DO $\mathrm{I}=1, \mathrm{~N}$

DO $\mathrm{J}=1, \mathrm{~N}$

$A(I, J)=W(I, J)$

$W(I, J)=0 . D 0$

ENDDO

$W(I, I)=P(I)$

ENDDO

RETURN

END

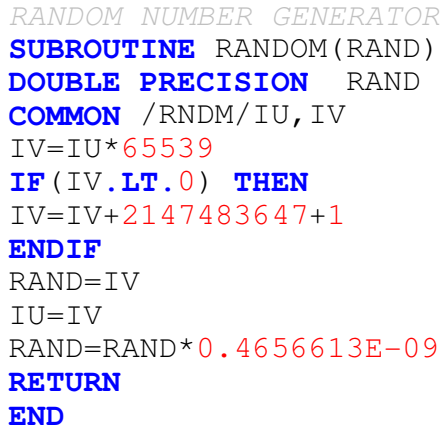

